\title{
Hitzeaktionspläne für Kliniken und Pflegeheime
}

\author{
Auf Temperaturextreme vorbereitet sein Systematisch erarbeitete und \\ verbindliche Konzepte schützen Patienten bzw. Bewohner und Beschäf- \\ tigte vor den gesundheitlichen Folgen von Hitzeperioden, die aufgrund \\ des Klimawandels häufiger werden. Warnungen des Deutschen Wetter- \\ dienstes müssen konkrete Schritte zur Folge haben, die dokumentiert und \\ evaluiert werden sollen. Langfristige Maßnahmen betreffen insbesondere \\ Bauvorhaben. \& Beate Blättner, Henny Annette Grewe und Debora Janson
}

\section{ZUSAMmenfassung}

Die steigende Häufigkeit und Intensität von Hitzeextremen ist mit gesundheitlichen Risiken verbunden. Vulnerable Gruppen sind Ältere, akut oder chronisch kranke oder beeinträchtigte Menschen, ungeborene Kinder, Säuglinge und Kleinkinder sowie Patienten, die bestimmte Medikamente einnehmen. In der Akutversorgung muss mit einer Erhöhung von Notfalleinweisungen gerechnet, in der Langzeitpflege erhöhter Mortalität vorgebeugt werden. Auch das Personal muss vor Hitze geschützt werden. Hitzeaktionspläne berücksichtigen langfristige wie auch Schutzmaßnahmen während der Hitzeperiode. Entsprechende Pläne können Teil des Qualitätsmanagements der Einrichtung sein. Obwohl eine Vorbereitung der Gesundheitssysteme politisch gefordert wird, gibt es in Deutschland bislang kaum Best Practice Beispiele.

\section{Schlüsselwörter: Hitzeextreme, Hitzeaktionsplan, Gesundheitsversorgung, Prävention}

\footnotetext{
D
} ie Hitzesommer 2003, 2006, 2010, 2015, 2018 und 2019 haben deutlich gemacht, dass es bereits jetzt in Deutschland sehr heiß sein kann. Schätzungen zufolge könnten aufgrund von Hitzeextremen künftig bis zu 8.500 Menschen pro Jahr zusätzlich sterben, wenn keine adäquate Anpassung an die Extremtemperaturen erfolgt. Neben Ländern und Kommunen sind insbesondere Gesundheitseinrichtungen gefordert, sogenannte Hitzeaktionspläne zu entwickeln. So hat die Sanitätsdirektion Wien 2018 einen Leitfaden für medizinische und pflegerische Einrichtungen zur Erstellung solcher Pläne herausgegeben. Die Bund-Länder Ad- hoc Arbeitsgruppe „Gesundheitliche Anpassung an die Folgen des Klimawandels (GAK)“veröffentlichte 2017 Handlungsempfehlungen zur Gestaltung von Hitzeaktionsplänen, in denen unter anderem Maßnahmenpläne für Alten- und Pflegeheime, Einrichtungen für Menschen mit körperlichen und geistigen Einschränkungen, Krankenhäuser, Not- und Rettungsdienste und Reha-Einrichtungen gefordert werden. Die Betreuungs- und Pflegeaufsicht Hessen legte für die Einrichtungen der Alten- und Behindertenhilfe bereits 2007 Informationen zur Vorbereitung auf Hitzeperioden vor, die 2017 überarbeitet wurden. Während des Sommers wird in hessischen Einrichtungen die Einhaltung der Maßnahmen überprüft.

Bundesweit stehen die Einrichtungen der Akut- und Langzeitpflege vor der gleichen Fragestellung: Wie können sie sich auf die kommenden Sommer vorbereiten, um den Schutz der Gesundheit auch während Hitzeperioden zu gewährleisten?

\section{Systematisch vorgehen}

Auf die jeweilige Einrichtung zugeschnittene Hitzeaktionspläne sind die Antwort auf diese Frage. Sie sollten unter Moderation des Qualitätsmanagements und Beteiligung der Heim- oder Klinikleitung sowie aller relevanten Bereiche entwickelt werden und zu verbindlichen Maßnahmen führen. Die Verantwortung für die Koordinierung liegt bei der Leitung. Ergänzend zur Entwicklung eines Aktionsplans kann es sinnvoll sein, ein Kapitel zur Hitze in medizinische und pflegerische Konzepte einzubinden. Idealerweise wird zunächst analysiert, inwieweit die Einrichtung betroffen ist:

- Befindet sich die Einrichtung in einer Region, in der mit einer hohen Anzahl an Hitzetagen zu rechnen ist (z.B. Oberrheingraben, städtisches Ballungszentrum)?

- Liegt die Einrichtung in einer besonders exponierten Lage mit reichlich versiegeltem Gelände oder ist sie in eine schützende Parkanlage mit schattenspenden Bäumen und kühlendem Wasser eingebettet? 


\section{PFLEGE MANAGEMENT}

_ Gibt es große Fensterflächen nach Süden oder Südwesten?

- Wie gut schützt die Bausubstanz vor hohen Innentemperaturen?

- Werden besonders vulnerable Personen versorgt (Kasten)?

- Wie empfinden die Beschäftigten die Arbeitsbedingungen an Hitzetagen?

- Sind an Hitzetagen höhere Arbeitsbelastungen beispielsweise aufgrund von zusätzlichen Notaufnahmen oder durch höheren Pflegeaufwand zu erwarten?

Der Analyse folgt eine Planung, die zwischen langfristigen und Akutmaßnahmen bei Hitze unterscheiden muss. Dabei wäre zu klären, ob für langfristige Maßnahmen eventuell eine finanzielle Förderung eingeworben werden kann. Zur Planung gehört auch zu identifizieren, ob Schulungen zur Sensibilisierung oder Festigung bereits vorhandener Kenntnisse sinnvoll sind. Festgehalten werden sollte zudem, wie die Wirksamkeit der Maßnahmen in Zukunft überprüft werden kann.

\section{Hitzewarnungen weitergeben}

Das Hitzewarnsystem des Deutschen Wetterdienstes (DWD) orientiert sich an der gefühlten Temperatur, einem bioklimatischen Maß für das thermische Wohlbefinden eines Modellmenschen. Zwei Warnstufen werden unterschieden: Starke Wärmebelastung (Warnstufe 1) tritt ein, wenn in einem Landkreis bzw. einer kreisfreien Stadt die gefühlte Temperatur an zwei Tagen in Folge über etwa $32^{\circ} \mathrm{C}$ steigen wird und nur eine geringe nächtliche Abkühlung erfolgt. Extreme Wärmebelastung (Warnstufe 2) ist mit einer gefühlten Temperatur über $38^{\circ} \mathrm{C}$ verbunden. Um entsprechende Informationen zu erhalten, kann man sich in einen Newsletter des DWD eintragen. Es bietet sich an, auch die Vorinformationen zu abonnieren, die bereits bis zu einer Woche vor der Hitzewarnung verschickt werden, damit eine bessere Vorbereitung möglich ist. Zudem sollten auch der Wärmeinseleffekt in Städten über 100.000 Einwohner*innen und der niedrigere Schwellenwert für Ältere (gefühlte Temperatur $36^{\circ} \mathrm{C}$ statt $38^{\circ} \mathrm{C}$ für eine extreme Belastung) mitkommuniziert werden.

Die hausinterne Weitergabe dieser Informationen erfolgt über eine verbindliche Informationskaskade, die im Hitzeaktionsplan festgelegt ist. Mit der Warnung sollten immer auch die wichtigsten Maßnahmen in Erinnerung gebracht werden, die umzusetzen sind.

\section{Räume kühl halten}

Die Reduktion der Exposition gegenüber Hitze in der Einrichtung und ihrer Umgebung ist das wichtigste Ziel der Schutzmaßnahmen. Die Temperatur in Innenräumen sollte $26^{\circ} \mathrm{C}$ nicht überschreiten. In Einzelfällen muss geprüft werden, ob ein Ausweichen in kühlere Räume möglich ist.

Um solche Temperaturen trotz Hitze zu erreichen, ist eine gute Isolation von Dach und Wänden erforderlich. Ein wichtiges Einfallstor für Hitze sind Fenster. Tagsüber geöffnete Fenster können es bei hohen Außentemperaturen in den Räumen nur heißer machen, denn die heiße Luft strömt nach innen. Wer Räume kühl halten will, muss in den Nacht- und frühen Morgenstunden lüften und tagsüber die Fenster geschlossen halten und beschatten. Ideal sind dafür Außenjalousien. Innenjalousien sind zwar finanziell meist günstiger als Außenjalousien, schützen aber weniger gut vor Hitze.

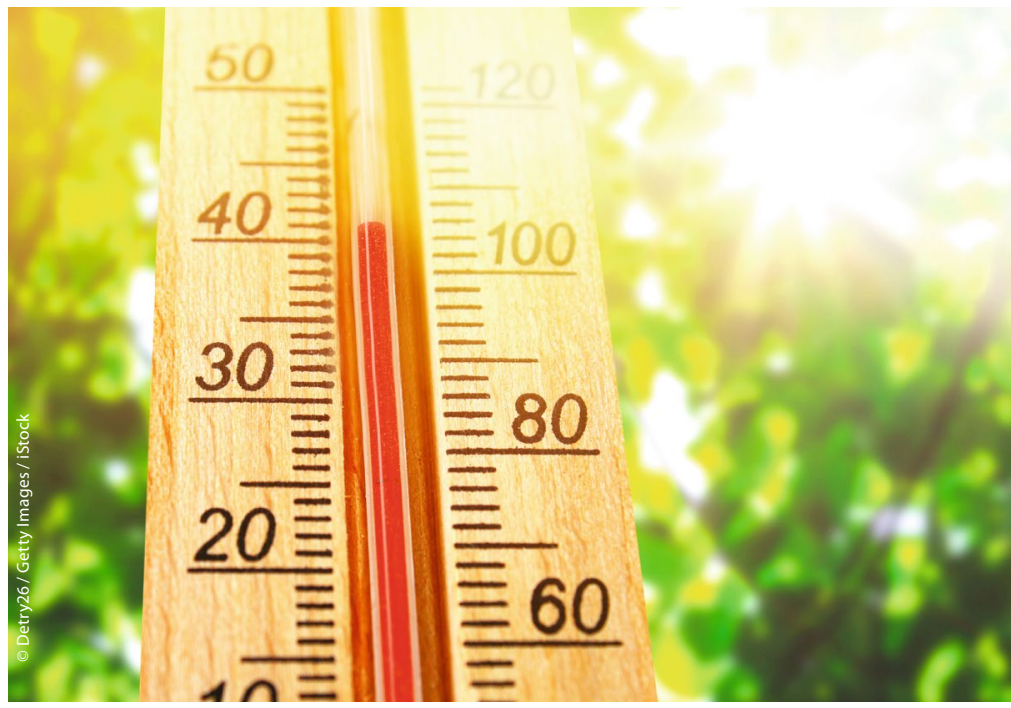

Kühlen Kopf bewahren: Hitzeaktionspläne schützen Mitarbeitende, Patienten und Bewohner.

\section{! GEFÄHRDETE PERSONENGRUPPEN}

Diese Menschen sind gegenüber Hitze besonders empfindlich:

_ Ältere Menschen über 75 Jahren

_ Ungeborene Kinder (Risiko Frühgeburt, Totgeburt), Säuglinge und Kleinkinder

- Menschen mit kognitiven oder körperlichen Einschränkungen

- Menschen mit akuten Erkrankungen wie akutes Nierenversagen, zerebrovaskuläre Krankheit, Herzversagen, Lungenentzündung, Infektionskrankheiten und Fieber

- Chronisch Erkrankte, insbesondere Menschen mit Herz-Kreislauf-Erkrankungen und Atemwegserkrankungen, Adipositas, Diabetes mellitus und anderen endokrinen Störungen, Nierenerkrankungen, psychischen und neurologischen Krankheiten wie Demenz, Alzheimer-Krankheit, Parkinson-Krankheit, Schizophrenie, wahnhaften Störungen, Depression, Störungen aufgrund von Alkohol- und Drogenmissbrauch

- Menschen, die folgende Medikamentengruppen einnehmen: Anticholinergika, Antipsychotika, Antihistaminika, Mittel gegen Parkinson, Antidepressiva, Anxiolytika und Mittel zur Muskelentspannung, Antiadrenergika und Betablocker, Sympathomimetika, Antihypertensiva und Diuretika, Antiepileptika, Antiemetika, Medikamente gegen Schwindel und gegen Urininkontinenz sowie Magen-Darm-Medikamente. 


\section{ARBEITSSCHUTZ}

$\mathrm{Ab} 30^{\circ} \mathrm{C}$ Innenraumtemperatur muss der Arbeitgeber Maßnahmen zum Schutz der Beschäftigten ergreifen. Bei über $35^{\circ} \mathrm{C}$ Raumlufttemperatur ist der Raum als Arbeitsraum ohne zusätzliche Schutzmaßnahmen nicht mehr geeignet. Die Arbeitsstättenregel (ASR) A3.5 führt detailliert auf, bei welcher Raumlufttemperatur welche Maßnahmen ergriffen werden sollen oder müssen. Grundsätzlich gilt: Der Arbeitgeber ist nach dem Arbeitsschutzgesetz zur Gefährdungsbeurteilung und zur Abwehr von Gefahren verpflichtet. Die ASR gilt in Pflegeheimen allerdings nicht, weil diese als Wohngebäude für Bewohner*innen und nicht als Arbeitsstätten zählen. Dennoch sind Arbeitgeber gehalten, auch dort für den Schutz von Beschäftigten zu sorgen.

\section{ERSTE HILFE BEI} HITZEBESCHWERDEN

Symptome und ihre Ursachen sind wie folgt:

- Hitzekrämpfe: Elektrolytmangel

- Hitzekollaps: Unterversorgung des Gehirns mit Sauerstoff durch Flüssigkeitsverlust und Blutdruckabfall

_ Hitzeerschöpfung: längerdauernder Verlust von Wasser und Mineralstoffen

- Hitzschlag: lebensbedrohlicher Anstieg der Körperkerntemperatur

Hitzeerschöpfung kann ohne Gegenmaßnahmen in einen lebensbedrohlichen Hitzschlag übergehen. Bei Hyperthermie über $39^{\circ} \mathrm{C}$, Verwirrungszustand oder anhaltendem Blutdruckabfall besteht Verdacht auf einen Hitzschlag!

Was bei Hitzebeschwerden zu tun ist:

_ in kühlere Umgebung bringen

_ hochlagern der Beine, damit das Blut besser in die Körpermitte fließen kann

_ kalte Umschläge auf Nacken und Stirn

_ oraler Elektrolyt- und Flüssigkeitsausgleich

- ggf. Massage krampfender Muskulatur bei Hitzekrämpfen

- Körpertemperatur messen. Bei erhöhter Temperatur in jedem Fall Ärztin oder Arzt hinzuziehen.

Denn: Fensterglas ist für den nicht sichtbaren Anteil von Sonnenstrahlen (kurzwellige Strahlen) gut durchlässig. Treffen sie auf im Raum befindliche Oberflächen werden sie als Wärmestrahlung (langwellige Strahlen) wieder abgegeben. Wärmestrahlung wird von Fensterglas zurückgehalten. Es entsteht eine Wärmefalle. Jalousien beschatten und wandeln auf ihrer Oberfläche kurzwellige Strahlen in langwellige Wärmestrahlen um. Passiert dies hinter dem Fenster (Innenjalousien), bleibt die Wärme zwischen Fenster und Jalousie, der Raum erwärmt sich. Geschieht es vor dem Fenster (Außenjalousien), kann innen kein Treibhauseffekt eintreten.

Auch Dach- und Fassadenbegrünung sowie Laubbäume in der Umgebung der Einrichtung können Innenräume kühler halten. Alle Möglichkeiten einer technischen passiven Kühlung sollten in Betracht gezogen werden.

Aktive Kühlung empfiehlt sich nur dann, wenn alle anderen Maßnahmen ausgeschöpft sind. Durch ihren hohen Stromverbrauch und die Abgabe von Wärme an die Außenluft tragen sie durch die weitere Aufheizung der Atmosphäre zum Klimawandel bei. Ähnliches gilt für raumbezogene Klimageräte, die zudem zur Unterkühlung führen können. Ventilatoren werden meist so eingesetzt, dass sie ihr Ziel nicht erreichen können: In einem geschlossenen Raum senken sie weder die Lufttemperatur noch die Luftfeuchte. Raumluftkühlung tritt nur dann ein, wenn beim beschleunigten Luftumsatz kühlere Luft anderen Ortes einströmen kann. Wenn es draußen aber wärmer ist als im Raum, kann dieser Effekt nicht eintreten. Eine erhöhte Luftströmung verstärkt zudem den mit Schwitzen verbundenen Wasser- und Elektrolytverlust, indem die mit Schweiß gesättigte Luft ständig von der Körperoberfläche entfernt wird und somit der Wasserdampfdruckgradient zwischen Haut und Umgebung erhalten bleibt.

Arbeitgeber sind im Rahmen des Arbeitsschutzes grundsätzlich verpflichtet, bei einer Raumtemperatur ab 30 Grad Maßnahmen zum Schutz der Mitarbeitenden zu ergreifen (Kasten).

\section{Behandlung und Pflege anpassen}

$\mathrm{Zu}$ den ärztlichen Aufgaben gehört es, die Medikation zu überprüfen und gegebenenfalls den Außentemperaturen anzupassen. Auch Vorgaben zur Trinkmenge sind zu überprüfen, dabei ist insbesondere auf gefährdete Personen mit erhöhten Risiken bei Hitzeextremen durch Hämokonzentration und Koronarthrombose, zerebrovaskuläre Ischämie und Niereninsuffizienz bzw. auf Personen mit Schlaganfall, Bluthochdruck, Diabetes, Koronarerkrankungen, Niereninsuffizienz oder Demenz zu achten. Der Elektrolythaushalt sollte überwacht und die Person auf Hinweise für Hitzebeschwerden beobachtet werden.

$\mathrm{Zu}$ den pflegerischen Aufgaben gehören die Überwachung der Körpertemperatur und die Kontrolle auf Anzeichen für eine Exsikkose (trockene Schleimhäute, stehende Hautfalten, konzentrierter, streng riechender Urin) oder Hitzebeschwerden (Kasten). Es ist auf leichte Bettwäsche und leichte Kleidung zu achten, synthetische Materialien können Hitzestau verursachen. Körperliche Anstrengung sollte nicht in den heißen Tagesphasen erfolgen. Es wird empfohlen, kühle (aber nicht kalte!) Waschungen oder Duschen anzubieten. Wichtig ist, hinreichend zu trinken; dabei ist aber das Risiko einer Hyponatriämie zu vermeiden, in dem auch hinreichend Salzhaltiges angeboten wird. Die Getränke sollten nicht gekühlt sein. Das Essen sollte sich auf mehrere kleine Mahlzeiten mit leichter Kost (viel Gemüse, wasserreiches Obst) verteilen. Wichtig ist zudem, die Temperatur in den Räumen zu überprüfen, in denen Medikamente gelagert werden. 
Aufgabe der Verwaltung ist es, die Temperatur in den Räumen zu kontrollieren und die Beschattungsfunktion sowie Belüftungsmöglichkeiten zu überprüfen. Dienstpläne sind ggf. dem höheren Arbeitsaufwand anzupassen. Auch für eine Umstellung der Essenspläne und für einen hinreichenden Getränkevorrat muss gesorgt werden. Es muss hinreichend leichte Bettwäsche vorhanden sein.

\section{Maßnahmen planen und evaluieren, Förderung prüfen}

Eine Evaluation der Maßnahmen beginnt mit der Dokumentation: Welche Raumtemperaturen wurden während der Hitzewelle tatsächlich erreicht? Welche Maßnahmen wurden faktisch umgesetzt und wo traten Schwierigkeiten auf? War ein erhöhter Arbeitsaufwand aufgrund von zusätzlichen Notfalleinweisungen oder zusätzlichem Pflegeaufwand nachweisbar? Welcher Veränderungsbedarf ergibt sich daraus?

Es kann sinnvoll sein, darüber hinaus nach harten Kriterien zu evaluieren: Ist die Komplikationsrate bzw. die Rate der Notfallverlegungen während der Hitzeperiode gestiegen? Gab es vermehrt Hinweise auf Hitzebeschwerden, Hyponatriämie oder Exsikkose? Ist die Anzahl an Todesfällen gestiegen? Welche zusätzlichen Maßnahmen müssen deshalb ergriffen werden?

In der Praxis dürften notwendige Baumaßnahmen zur Verbesserung des Hitzeschutzes oft die größten Hindernisse bei der Umsetzung eines Hitzeaktionsplans für Heime und Kliniken darstellen. Hier kann das Förderprogramm „Klimaanpassung in sozialen Einrichtungen“ des Bundesministeriums für Umwelt, Naturschutz und nukleare Sicherheit (BMU) gegebenenfalls Unterstützung bieten. Es hat ein Fördervolumen von 150 Millionen Euro. Krankenhäuser, Pflege- und Altenheime sowie Hospize sind explizit antragsberechtigt.

Die Umsetzung der weiteren Maßnahmen ist im Einzelnen vergleichsweise einfach. Maßnahmen müssen aber immer in ein Gesamtkonzept der Einrichtung eingebettet sein. Dies zu entwickeln braucht Zeit und die Beteiligung möglichst aller Betroffenengruppen.

\section{FAZIT}

Gesundheitseinrichtungen sind, neben Ländern und Kommunen, gefordert, sogenannte Hitzeaktionspläne zu entwickeln, um auf Temperaturextreme vorbereitet zu sein und Mitarbeitende, Patienten und Bewohner zu schützen.

Aktive Kühlung empfiehlt sich nur, wenn alle anderen Maßnahmen ausgeschöpft sind.

Zu den pflegerischen Aufgaben gehören bei Hitzeperioden die Überwachung der Körpertemperatur und die Kontrolle auf Anzeichen für eine Exsikkose oder Hitzebeschwerden.

Eine umfangreiche Literaturliste finden Sie im eMagazin der PflegeZeitschrift und auf springerpflege.de.

\section{Autorenkontakt:}

Prof. Dr. phil. Beate Blättner forscht seit 2008 im Public Health Zentrum der Hochschule Fulda zu den Auswirkungen des Klimawandels auf die menschliche Gesundheit und zu Präventionsansätzen. Ihr Schwerpunkt sind dabei Hitzeextreme und die Implementierung von Hitzeaktionsplänen. E-Mail: Beate.Blaettner@pg.hs-fulda.de.

Prof. Dr. med. Henny Annette Grewe, forscht ebenfalls seit 2008 im Public Health Zentrum der Hochschule Fulda zum gleichen Forschungsgebiet.

Debora Janson, M.A., ist wissenschaftliche Mitarbeiterin an der Hochschule Fulda, Fachbereich Pflege und Gesundheit, und arbeitet seit 2019 in der Arbeitsgruppe.

\section{Hier steht eine Anzeige.}

